RESEARCH ETHICS

\title{
How to handle informed consent in longitudinal studies when participants have a limited understanding of the study
}

\author{
G Helgesson, J Ludvigsson, U Gustafsson Stolt
}

See end of article for

authors' affiliations

J Med Ethics 2005;31:670-673. doi: 10.1136/jme.2004.009274

Correspondence to:

Gert Helgesson, PhD in

philosophy, Researcher at

the Centre for Bioethics at

Karolinska Institutet and

Uppsala University,

Sweden; Gert.Helgesson@

bioethics.uu.se

Received 6 May 2004

In revised form

24 January 2005

Accepted for publication

19 February 2005

\begin{abstract}
Empirical findings from a Swedish longitudinal screening study show that many of the research subjects had a limited understanding of the study. Nevertheless they were satisfied with the understanding they had and found it sufficient for informed continued participation. Were they wrong? In this paper, it is argued that the kind of understanding that is morally required depends partly on the kind of understanding on which the research subjects want to base their decisions, and partly on what kind of knowledge they lack. Researchers must ensure that the information process is not flawed and that participants receive the information they want. To achieve this, new information efforts may be needed. Researchers must also ensure that research subjects have knowledge about aspects of importance to them. Lack of understanding may, however, be the result of conscious choices by research subjects to disregard some of the information because it is not important to them. Such choices should normally be respected.
\end{abstract}

11 nformed consent" is a central concept in bioethics and the practice of obtaining informed consent is an integral part of medical research. In order to ensure that consent procedures are more than empty ritual, researchers must be clear about what is needed for consent to be sufficiently informed in various concrete situations. An often mentioned prerequisite for morally relevant consent is that research subjects have some genuine understanding of the study in which they participate. Yet empirical studies show that participants in clinical research sometimes have limited understanding due, for instance, to inadequate information, misunderstandings, and ignorance of options, and of potential risks and benefits. ${ }^{1-3}$

Empirical data generated from a case study within the Swedish All Babies in Southeast Sweden (ABIS) study, an ongoing longitudinal cohort study on the importance of environmental factors for development of Type 1 diabetes, support this finding. Moreover, they reveal an interesting discrepancy. On the one hand, an overall majority of the participants report that they are satisfied with the information, understand it, regard it as sufficient for decision making, and have made a completely voluntary decision to participate. ${ }^{4}$ On the other hand, observations indicate that although the participants have a fair understanding of the research methods, their knowledge and comprehension of aim, purpose, and potential benefits is limited. ${ }^{5}$ There is therefore, prima facie, reason to doubt that all participants have a morally sufficient understanding of the study.

In order to judge whether they do, we must know not only what understanding they have, but also what the criteria for a morally sufficient understanding are. The latter can only be clarified by ethical analysis.

In this paper, we take empirical data as a starting point for a discussion of what is required for "sufficient understanding". On the basis of this analysis, we suggest and argue for ways to handle situations where research subjects have limited understanding of the study in which they are participating.

\section{EMPIRICAL BACKGROUND}

The empirical material concerns the ABIS study-a longitudinal cohort study. The cohort sample comprises all births in a defined geographical area in a two year period (October 1997-October 1999). The general aim of ABIS, which will continue until year 2013, is to study the interaction between multiple genes and gene environment with regard to the development of Type 1 diabetes (IDDM) and other multifactorial diseases such as allergies, rheumatism, inflammatory bowel disease, and cancer. More specific aims are identification of high risk children and potential prevention of Type 1 diabetes. Methods include analysis of biomaterial (blood samples, breast milk, hair, stools, urine), a diary covering the child's first year, and intermediate questionnaires (at birth and at one, two, five, eight, eleven and fourteen years). Initially, of those asked to participate, $78.6 \%$ agreed, which means that data from 17005 children are included in the ABIS study.

The ABIS consent procedures include oral and written information, which is given during pregnancy and at the time of birth. An information video was also produced to be viewed during visits to the maternity welfare centre. In addition, written information is offered at every control visit. Potential participating parents are informed about the research group, the design and aims of the study, future intermediate questionnaires, and what samples it is planned to take and store. They are also told that biological and environmental factors involved in the development of multifactorial diseases will be mapped, that certain specified analyses (including genotyping and autoantibodies analysis) will be made in order to predict high risk individuals, and that prevention of diabetes will be offered if and when effective methods become available.

The data presented here were generated from two "checkpoints" with a four year interval between them:

1. a questionnaire to 400 randomly chosen participant families after one year of participation in the ABIS study (73.3\% answer rate),

2. pilot data from 2900 respondents participating in the ABIS study's regular five year questionnaire.

The results from the first checkpoint, an independent research ethical case study, showed that the majority of the participants 
- were satisfied with the information provided;

- regarded it sufficient for them to make their decisionthat is, to give informed consent to participate in the ABIS study;

- regarded themselves as having understood the information they received, and

- regarded themselves as having made a completely voluntary decision. ${ }^{4}$

On the other hand, the findings also indicate a considerable gap between the proportion of participants who reported understanding of, and satisfaction with, the information provided and the proportion who were either unsure of, or disagreed with, several of what may be called the basic aims and methods of the ABIS screening. Most noticeable are the findings regarding identification of high risk children, potential future contacts and preventions/interventions, and future intermediate questionnaires. ${ }^{5}$ Although $68 \%$ acknowledged the aim to identify children at high risk for Type 1 diabetes, $19 \%$ disagreed that this was an aim, and 13\% were not sure. Less than half of the respondents (45\%) were aware that they might be contacted if their child was identified as belonging to the high risk group. Twenty five per cent stated that they were aware of, or recalled, that if prevention were possible in the future it might be carried out. Thirty eight per cent of the respondents stated that they were aware that they would be asked to complete questionnaires in the future. ${ }^{5}$

Questions similar to those in the first questionnaire were incorporated into the ABIS study's regular five year questionnaire. The new findings are fairly consistent with the earlier results. Although $83 \%$ acknowledge the aim of identifying children in the birth cohort with a high risk of developing Type 1 diabetes, only $48 \%$ of the participants state that they are aware that they might be contacted during the study if their child is identified as belonging to the high risk group. Thirty three per cent state that they are aware that there could be future intervention or prevention. Despite these facts, $78 \%$ report having sufficient knowledge for continued participation at the time of completing the questionnaire.

\section{WAYS TO INCREASE UNDERSTANDING}

Our empirical data show that participants in ABIS have limited knowledge of certain aspects of the project. What should researchers do in such a situation? We argue, from the assumption that autonomy and personal integrity are of central ethical importance when answering this question, that what the appropriate action is depends partly on participants' attitudes toward their lack of understanding, and partly on what kind of knowledge they lack.

"Autonomy", as used here, refers to self determination in the sense of making one's own decisions based on one's own values, and an understanding of the situation at hand, without undue interference by others. Since understanding, self reflection, and absence of interfering influences come in degrees, autonomy is also a matter of degrees. There are distinctions to be made between the capacity to act autonomously, the actual condition of acting autonomously, and the ideal of character derived from that conception. The capacity for autonomous decision making is a precondition of genuine informed consent, and the use of procedures aimed at ensuring informed consent is, in turn, a way to facilitate autonomous action..$^{6-11}$ Since autonomy is about individuals deciding for themselves about their own lives, it is closely connected to personal integrity. Central to personal integrity is the idea of (a right to) a personal sphere. Respecting a person's integrity involves respecting her privacy and in particular her understanding of her own life. ${ }^{12}$
A natural reaction to empirical findings demonstrating a lack of understanding among research participants, such as ours, is that the information process is flawed and that something must be done to correct it. It may indeed be the case that people lack understanding because of poor information: it may be too sparse or too complicated, or research participants may not be given a satisfactory opportunity to ask questions and have their personal worries and interests addressed. However, if people have a limited understanding of an ongoing study, this does not necessarily mean that they did not have that understanding when they decided to participate, which was perhaps several years agoforgetfulness is a plausible explanation. In either case research subjects are left with limited knowledge of the study in which they are participating. ${ }^{13}$

If research participants feel their understanding is insufficient, as $22 \%$ of the ABIS participants stated in the five year questionnaire, then something must be done, even if (as in the ABIS case) all participants had previously been informed that they could ask for any information they wanted. Before taking new steps to improve information, researchers should ensure that the information they are providing is relevant, unbiased, and expressed in language simple enough to be easily understood. Excessive detail should be avoided, because it can contribute to confusion rather than enhance understanding. Potential participants may also be discouraged by overly long information sheets, which may be the result of researchers trying to "cover everything" or designing the information primarily to meet medicolegal requirements. Repeating, summarising, and presenting information in easily absorbed "portions" are useful strategies to avoid confusion when information is presented. Other recommendations are: ${ }^{14}$

- Use language that reflects the participants' autonomy (to underline that the decision to participate, or opt out, is still theirs);

- Check their understanding, for instance, by letting them summarise pieces of information in their own words;

- Invite questions and comments;

- Provide time and opportunity to discuss their concerns thoroughly, and

- Explicitly offer ongoing support should they wish to ask further questions in the future.

Even if research participants are satisfied with a limited understanding of the study, researchers should try to increase understanding if what is at stake is sufficiently important. This is the case, for instance, if participation involves considerable risk of harm or affects participants' basic capabilities to maintain, or achieve, an acceptable degree of autonomy and personal integrity (Dworkin, ${ }^{7}$ pp 114, 118, 119).

\section{UNDERSTANDING IN THE INTEREST OF PARTICIPANTS}

Although legal regulations and ethical guidelines often claim quite advanced understanding to be necessary for informed consent, a basic understanding, which includes that the person understands that she is being asked to participate in a research study, that it is up to her to decide, that she has an unrestricted right to withdraw consent at any time, and the expected benefits and costs (in terms of risks and time) involved, is often sufficient from an ethical perspective. ${ }^{6}{ }^{15}$

Of major importance when judging how to handle situations where research subjects have a limited understanding is how the lack of understanding relates to what is important to them. It is pointless to put a lot of effort into ensuring that they have a genuine understanding of those 
aspects of the study that are mainly of interest to researchers, such as information on specific methods used in the study. Admittedly, if "methods" is interpreted as referring to practical procedures that affect research subjects, then information on methods is indeed required. If, however, "methods" refers to scientific methods, then such information widely exceeds what most research subjects would ever care, or need, to know.

Much is gained if the information requirement is not seen as being a requirement for a specific and "fixed" information package, but rather is seen as representing a duty to help research subjects understand what they are involved in (and to help potential research subjects understand what they are being asked to get involved in).

Most of what is written on informed consent, both in academia and general debate, focuses on the entitlements participants have to information. This is certainly important. Information relevant to people's decisions whether or not to participate in research, or to withdraw from an ongoing study, must not be withheld. The problem of what to do if research subjects want less information than what might seem needed for thoroughly informed consent has, however, been mainly ignored. This problem is particularly acute in modern society, with its abundance of information, where people get by only by disregarding much of what washes over them. Any practical handling of information to potential or actual research subjects must be sensitive to the fact that people may want to know very little or a great deal. Respect for autonomy requires not only that participants are given the opportunity to act on information concerning the study but it also requires that the amount of information they receive is adjusted to the amount of information they want.

When researchers do not know why participants lack understanding of the study, they need to find out if this is voluntary or involuntary. A lack of understanding may be a result of a conscious choice not to process all the information offered. This presents a delicate problem to those responsible for the study: in order to know how to handle such a situation, they must know why participants lack understanding. If the reason is that (some or all) participants want limited information only, this may be because they want as little interference in their lives as possible. Then to be asked whether they are happy with the understanding they have would be an additional unwanted interference. This is not to mention how they would feel about having information forced on them until researchers felt the point had been reached where they needed no more. Indeed that point might never be reached if researchers insist that participants in their study should have certain knowledge that some or all of these participants do not want. To avoid this problem, researchers need to know beforehand what attitudes toward information and privacy different participants have. If possible, they should find this out at the onset of the study otherwise participants should be offered an opportunity to get more detailed information in addition to the initial information given.

\section{SUFFICIENT UNDERSTANDING FOR INFORMED CONSENT}

As we have seen, participants in the ABIS screening project had limited knowledge of some basic aspects of that project. We have argued that limited understanding is not necessarily a problem that has to be met with additional information. It could, however, be argued that nevertheless it is a problem because participants' understanding could be considered insufficient for morally satisfactory informed consent. Beauchamp and Childress, in their influential Principles of Biomedical Ethics, claim that the following requirements must be satisfied for morally satisfactory informed consent (Beauchamp et al, ${ }^{13}$ p 80):

1. competence (to understand and decide),

2. voluntariness (in deciding),

3. disclosure (of material information),

4. understanding (of 2 and 3),

5. decision, and

6. authorisation ${ }^{\mathrm{i}}$.

For research purposes, an informed consent (or refusal) is morally satisfactory, according to these requirements, if and only if the research subject is competent to understand information about the study and about possible risks and benefits for herself; is in a position to make a voluntary (unforced) choice; receives and understands relevant information; makes a decision, and in some form authorises or refuses to authorise the researchers to include her in the study. Also she must be aware that her consent constitutes such an authorisation. Information and consent procedures aim at providing the potential research subject with an opportunity to make an autonomous choice. In order to choose autonomously the person must understand what the choice concerns, the basic facts about the study, and how it affects participants. Although some facts may be vital for this decision, others are not. Potential participants must be adequately informed, but they do not have to be fully informed, based on the information they need to form an adequate understanding — an adequate understanding being a precondition for an autonomous choice. A full understanding is not necessary (Beauchamp et al, ${ }^{13}$ pp 77, 88-90) (O'Neill, ${ }^{15}$ especially pp 42-4, 156-7).

Unfortunately, this does not tell us how much research subjects have to know in order for their understanding to be sufficient for morally satisfactory consent. Do they need all the information they would consider relevant to their choice (if they had it), or is it acceptable that they base their decision on less than that? This becomes crucial whenever (potential) research subjects want to process only some of the relevant information because they have other priorities.

\section{RELEVANT INFORMATION MAKES A DIFFERENCE}

The idea that all relevant information must be understood may seem appealing, because this means research participants have more control of their choices. Choices based on all relevant information are more likely to be consistent with what participants really want-and therefore to be more authentic - than choices based on less than that (Feinberg, ${ }^{9}$ pp 32-3). It may be argued, however, that this view of autonomy does not sufficiently consider the importance of self management - that is, of deciding for oneself and acting on these decisions without interference. The "all relevant information" view of autonomy may conflict with the self management view since the self managing agent may not want to base her decision on all relevant information. The question of what is an autonomous choice is thus answered in different ways by the two positions: is it (i) the choice that the agent willingly makes, based on the information she wants, or (ii) the choice she would make if she had all the relevant information (even if, in the present situation, she does not have all the relevant information, does not want it, and does not want to make the choice she would then have made)?

We cannot hope to settle this dispute here. Both positions share the view, however, that the information should make a

'Their list contains a seventh point: "recommendation (of a plan)", which mainly concerns doctor/patient relations where a certain treatment is recommended in a healthcare situation. 
difference to the choices participants make, or would make, if it is to be regarded as needed for a morally satisfactory informed consent. Assume, for example, that Anne has consented to participate in a study on cardiovascular diseases. She has received clear, well written information and a medical doctor has patiently and carefully explained everything she wants to know about the study, and quite a bit more as well. She did not understand the explanations about the scientific methods that will be used, but she does not care because the doctor has assured that they are as safe as previous methods. Furthermore, she did not listen very attentively when the doctor explained in some detail what new knowledge they hope to gain from the study. What matters to her is that the researchers appear to have well founded hopes that they will get important and useful results. ${ }^{16}$ In this case, knowledge about scientific methods and details of research aims are not needed for a morally satisfactory informed consent from Anne. This does not alter the fact that the very same information can be relevant and needed for other potential research subjects' consent (or refusal). There is, however, no reason to maintain that everyone participating in the study needs to understand this information, because it is not relevant to every participant's choice.

One reason why research participants sometimes show a limited interest in detailed information might be that they trust the researchers and the research community. ${ }^{17}$ Through the regular use of clear and concise information and consent procedures - that is, by openly presenting the facts and letting people decide for themselves-the research community can prove itself to be trustworthy. If the research community is trusted, then participants may feel happy to disregard most of the information offered and settle for some basic facts about how their participation will affect them in practice. ${ }^{11}{ }^{15}$ That ABIS participants trust the ABIS research group is indicated in our empirical data, which shows (1) that an overwhelming majority of the participants have a positive attitude toward the ABIS study and feel comfortable about participating, and (2) that they say "yes" to having their personal data correlated to official registers if that is needed for research purposes.

\section{CONCLUSIONS}

A number of studies show that sometimes research participants lack understanding of the study in which they participate. Our own empirical data show this may be the case even if participants report both being satisfied with the information they have received and finding it sufficient for their informed consent to participate. Although $78 \%$ of those who responded to the five year questionnaire of the ABIS study said they had sufficient understanding of the study, $52 \%$ were unsure about or disagreed with the statement that they might be contacted during the study if their child were identified as belonging to the high risk group for Type 1 diabetes. Sixty seven per cent did not correctly state, or could not say for certain, that preventive action could be taken in the future for children belonging to the high risk group.

There are different explanations as to why there should be such a discrepancy between measured levels of understanding and research subjects' attitudes toward their understanding. Participants may be satisfied even though the information process is flawed-that is, they may be satisfied with the information they have even if relevant pieces of information are lacking or the information has not been successfully communicated. Another possibility is that although participants no longer remember the information provided, they remember being satisfied with it. If the information was deficient, then new efforts at providing information are needed for there to be informed consent.

Lack of understanding is not, however, necessarily a result of insufficient routines for supplying information. It may also be the result of a conscious choice on behalf of research subjects to disregard some of the information. If this is the case, then renewed information efforts can be expected to have little effect, apart from possibly disturbing those who have chosen to base their decision to participate on a more limited amount of information. Such a choice should, in most cases, be respected. This presents researchers with a problem, because in many cases they cannot be certain why participants lack understanding: is it because of failures in communication or because of conscious choices on the part of the participants? Methods to identify people in the latter category are needed. These must not, however, interfere any more than necessary with these people's lives.

\section{Authors' affiliations}

G Helgesson, The Centre for Bioethics at Karolinska Institutet and Uppsala University, Sweden

J Ludvigsson, The Division of Paediatrics at the Department of Molecular and Clinical Medicine, Linköping University, Sweden

U Gustafsson Stolt, The Division of Paediatrics at the Department of Molecular and Clinical Medicine, Linköping University, Sweden

\section{REFERENCES}

1 Giardello FM, Brensinger MS, Petersen GM, et al. The use and interpretation of commercial APC gene testing for familial adenomatous polyposis. N Engl J Med 1997;336:823-7.

2 Daugherty CK. Impact of therapeutic research on informed consent and the ethics of clinical trials: a medical oncology perspective. J Clin Oncol 1999;17:1601-17.

3 Joffe S, Cook EF, Cleary PD, et al. Quality of informed consent: a new measure of understanding among research subjects. J Nat Cancer Institute 2001;93:139-46.

4 Gustafsson Stolt U, Ludvigsson J, Liss P-E, et al. Bioethical theory and practice in genetic screening for type 1 diabetes. Med Health Care Philos 2003;6:45-50

5 Gustafsson Stolt U, Helgesson G, Liss P-E, et al. Information and informed consent in a longitudinal screening involving children: a questionnaire survey. Eur J Hum Genet 2004;13:376-83.

6 Brock D. Life and death: philosophical essays in biomedical ethics. Cambridge: Cambridge University Press, 1993, ch 1.

7 Dworkin G. The theory and practice of autonomy. New York: Cambridge University Press, 1988, especially chs 1 and 7.

8 Faden R, Beauchamp T. A history and theory of informed consent. Oxford: Oxford University Press, 1986.

9 Feinberg J. Autonomy. In: Christman J, eds. The inner citadel. Essays on individual autonomy. Oxford: Oxford University Press, 1989:27-53.

10 Meyers C. Cruel choices: Autonomy and critical care decision making. Bioethics 2004; 18:104-19.

11 Stiggelbout AM, Molewijk AC, Otten W, et al. Ideals of patient autonomy in clinical decision making: a study on the development of a scale to assess patients' and physicians' views. J Med Ethics 2004;30:268-74.

12 Dahl Rendtorff J. Basic ethical principles in European bioethics and biolaw: autonomy, dignity, integrity and vulnerability-towards a foundation of bioethics and biolaw. Med Health Care Philos 2002;5:235-44 (at 243).

13 Beauchamp T, Childress R. Principles of biomedical ethics [5th ed]. New York: Oxford University Press, 2001:89-90.

14 Brown RF, Butow PN, Butt DG, et al. Developing ethical strategies to assist oncologists in seeking informed consent to cancer clinical trials. Soc Sci Med 2004;58:379-90 (especially 380, 383, 385).

15 O'Neill O. Autonomy and trust in bioethics. Cambridge: Cambridge University Press, 2002.

16 Hoeyer K. "Science is really needed-that's all I know": informed consent and the non-verbal practices of collecting blood for genetic research in Sweden. New Genet Soc 2003;22:198-212.

17 Busby H. Blood donation for genetic research. What can we learn from donors' narratives? In, Tutton R, Corrigan O, eds. Genetic databases: Socioethical issues in the collection and use of DNA. London: Routledge, 2004:39-56 (at 47). 